\title{
Predicted osteotomy planes are accurate when using patient-specific instrumentation for total knee arthroplasty in cadavers: a descriptive analysis
}

\author{
A. J. Kievit ${ }^{1,2}$ • J. G. G. Dobbe ${ }^{3}$ G. J. Streekstra ${ }^{3}$ L. Blankevoort ${ }^{1}$ M. U. Schafroth ${ }^{1}$
}

Received: 22 March 2017 / Accepted: 14 September 2017 / Published online: 25 September 2017

(c) The Author(s) 2017. This article is an open access publication

\begin{abstract}
Purpose Malalignment of implants is a major source of failure during total knee arthroplasty. To achieve more accurate 3D planning and execution of the osteotomy cuts during surgery, the Signature (Biomet, Warsaw) patient-specific instrumentation (PSI) was used to produce pin guides for the positioning of the osteotomy blocks by means of computeraided manufacture based on CT scan images. The research question of this study is: what is the transfer accuracy of osteotomy planes predicted by the Signature PSI system for preoperative 3D planning and intraoperative block-guided pin placement to perform total knee arthroplasty procedures? Methods The transfer accuracy achieved by using the Signature PSI system was evaluated by comparing the osteotomy planes predicted preoperatively with the osteotomy
\end{abstract}

A. J. Kievit

a.j.kievit@amc.uva.nl

J. G. G. Dobbe

j.g.dobbe@amc.uva.nl

G. J. Streekstra

g.j.streekstra@amc.uva.nl

L. Blankevoort

1.blankevoort@amc.uva.nl

M. U. Schafroth

m.u.schafroth@amc.uva.nl

1 Orthopaedic Research Center Amsterdam, Department of Orthopaedic Surgery of the Academic Medical Center, University of Amsterdam, Amsterdam Movement Sciences, 1100 DE Amsterdam, The Netherlands

2 Department of Orthopedics of the Tergooi hospital, Hilversum, The Netherlands

3 Department of Biomedical Engineering and Physics of the Academic Medical Center, University of Amsterdam, 1100 DE Amsterdam, The Netherlands planes seen intraoperatively in human cadaveric legs. Outcomes were measured in terms of translational and rotational errors (varus, valgus, flexion, extension and axial rotation) for both tibia and femur osteotomies.

Results Average translational errors between the osteotomy planes predicted using the Signature system and the actual osteotomy planes achieved was $0.8 \mathrm{~mm}( \pm 0.5 \mathrm{~mm})$ for the tibia and $0.7 \mathrm{~mm}( \pm 4.0 \mathrm{~mm})$ for the femur. Average rotational errors in relation to predicted and achieved osteotomy planes were $0.1^{\circ}\left( \pm 1.2^{\circ}\right)$ of varus and $0.4^{\circ}\left( \pm 1.7^{\circ}\right)$ of anterior slope (extension) for the tibia, and $2.8^{\circ}\left( \pm 2.0^{\circ}\right)$ of varus and $0.9^{\circ}\left( \pm 2.7^{\circ}\right)$ of flexion and $1.4^{\circ}\left( \pm 2.2^{\circ}\right)$ of external rotation for the femur.

Conclusion The similarity between osteotomy planes predicted using the Signature system and osteotomy planes actually achieved was excellent for the tibia although some discrepancies were seen for the femur. The use of 3D system techniques in TKA surgery can provide accurate intraoperative guidance, especially for patients with deformed bone, tailored to individual patients and ensure better placement of the implant.

Keywords Total knee arthroplasty · Biomet Signature, patient-specific instrumentation $\cdot$ Accuracy study $\cdot 3 \mathrm{D}$ Analysis $\cdot \mathrm{CT}$

\section{Introduction}

Malalignment or an incorrectly sized implant is the major cause of failure in total knee arthroplasty (TKA) [12]. In conventional TKA preoperative planning, patients are assessed on the basis of standing anteroposterior and lateral radiographs, sunrise view of the patella or standing wholeleg radiographs to determine the mechanical and anatomical 
axis. The aim of preoperative planning and assessment of the tibia and femur is to determine the quality of bone stock, to estimate correct relative axial rotational and translational alignment and the position of the joint line and also to select a correctly sized implant. The additional benefits of accurate planning are shorter operation times and reduced risk of complications.

Until recently, preoperative planning based on 2D radiographs was the recommended method to prepare for total knee arthroplasty (TKA). However, recent studies have shown that $2 \mathrm{D}$ preoperative methods are not always reliable for TKA $[1-3,14,16]$. More accurate 3D computer-assisted techniques are now being employed; for example, the navigation techniques in computer-assisted surgery (CAS) help increase alignment accuracy $[5,9]$.

Likewise, 3D patient-specific instrumentation (PSI) systems are increasingly used in preoperative planning for TKA to predict the alignment of osteotomy planes. Currently, nine commercial PSI systems are available for use in knee arthroplasty procedures [19] of which the Signature ${ }^{\mathrm{TM}}$ Personalized Patient Care system (Biomet Signature Knee System: in collaborative partnership with Materialise NV) is most commonly used [25]. The Signature technique processes data from preoperative CTs or MRIs of patients' entire lower limbs to produce patient-specific guides that match each individual's anatomical geometry. These patientspecific guides ensure optimal placement of the stainless steel mechanism guiding the oscillating saw that cuts the planes in tibia and femur. The aim of patient-specific guides is to improve the accuracy between predicted and achieved osteotomy planes and thus reduce operation time and the risk of complications. Moreover, this technique does not cause intramedullary damage, in theory, reducing the risk of fat embolisms [13] although this claim has not yet been proven. A further advantage of such a system is for use in patients where standard anatomical landmarks are unreliable because of bone deformation caused by (iatrogenic) trauma or developmental problems.

The added value of PSI has been questioned in recent studies $[4,6,18,23]$, even though more than 80,000 PSIassisted operations were performed in 2012 worldwide [25]. Many of these recent PSI studies only looked at the final position of the implant as a measure of success. However, implant position does not necessarily indicate that the optimal osteotomy plane was actually achieved as the cement used can obscure the planes. It is clear that to justify the use of PSI, the prediction and orientation of achieved osteotomy planes should be better, or at least as accurate, as conventional 2D systems reported in the literature.

Therefore, this study aims to show the added value of a $3 \mathrm{D}$ based system for predicting the position and orientation of osteotomy planes preoperatively in individual patients so as to provide accurate intraoperative guidance and ensure better placement of the implant and a greater chance of recovery.

\section{Materials and methods}

\section{D prediction, planning and surgery}

The preoperative prediction study was performed using CT images of nine fresh-frozen whole human legs (foot to femur head) as data for the 3D Signature Personalized Patient Care software (Biomet, Warsaw, USA). The specimens had a median age of 82 years (min-max 71-92; six males and three females; six right and three left limbs). The CT was chosen for scanning, as images are considered more accurate than those from MRI [26]. CT scans were made using a Brilliance 64-channel CT scanner (Philips Healthcare, Best, the Netherlands). A clinical scanning protocol was used to make the CT scans for both the planning and evaluation of the actual cutting planes. The osteotomies of interest were the distal femur cut, the posterior femur cut and the proximal tibia cut as they are made using pin placement via the guides. The femur osteotomy planes were predicted using the Signature software with zero degrees of extra varus/ valgus (coronal projection) adjustment along the anatomical axis, three degrees of flexion (sagittal projection) in the femur and zero degrees of rotation in the axial projection. The tibia osteotomy was predicted at a standard zero degree of varus/valgus and zero degrees of posterior slope. Subsequently, the researchers sent the CT data sets via Biomet to Materialise (Leuven, Belgium), who manufactured the specific femur and tibia guides and sent these to the surgeon.

A single surgeon, with extensive experience in TKA surgery, carried out the surgical procedures. A standard medial parapatellar approach was used to expose the femur and tibia. Firstly, the tailor-made femur guide was positioned correctly in relation to the supplied 3D bone model. Once the surgeon was satisfied with the guide placement, the guide was fixed in place with four pins (Fig. 1). Subsequently, the cutting guide for the surgical saw was slid over the pins and the osteotomies were performed. Three planes were used for evaluation, i.e. the distal femur cut, the posterior femur cut and the proximal tibia cut (Fig. 2).

To ensure that the methods and surgery were comparable and executed as intended, all osteotomies were planned and performed in the same hospital using the Signature software.

\section{Comparing planned osteotomy planes with actual planes}

To evaluate the postoperative osteotomies, another CT scan was made after the osteotomies had been performed. A validated and accurate method was used for assessment of the 
Fig. 1 Pin-positioning guides snugly fit to the proximal tibia in lateral view (T1) and anteroposterior view (T2). The femoral guide is shown from distal to proximal, above being the anterior femur $(\mathrm{F} 1)$, and from anterior to posterior, above being the distal femur (F2)
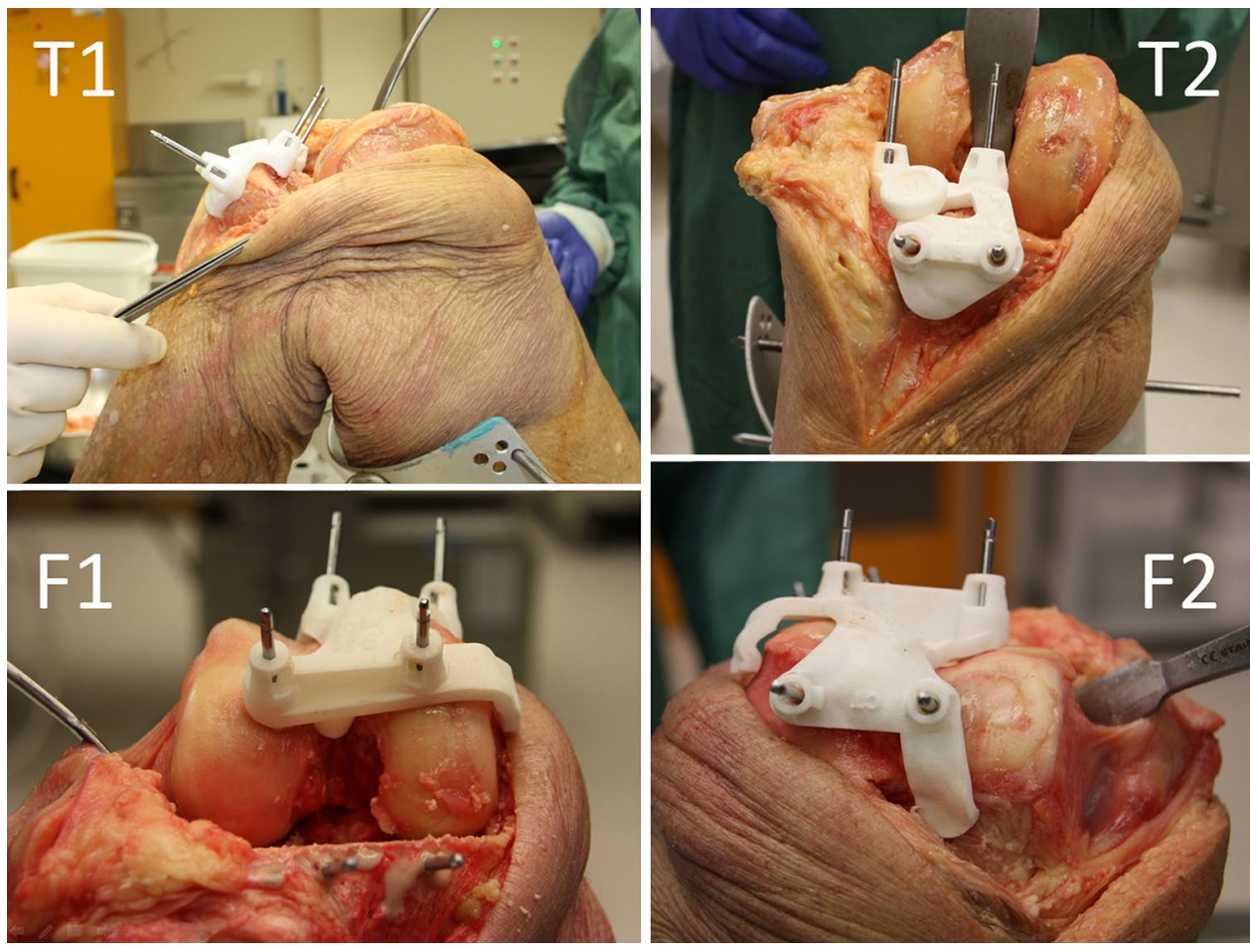

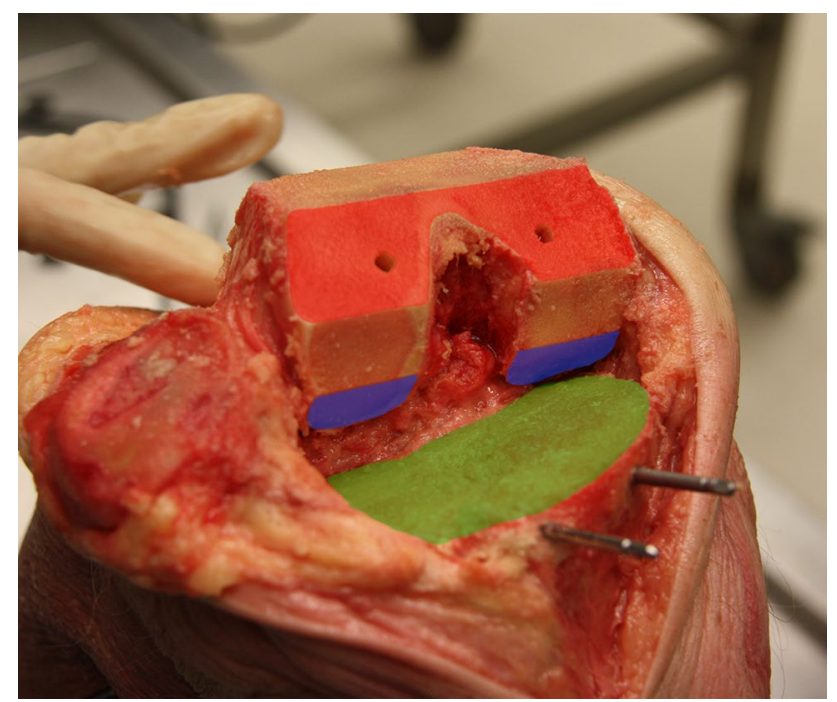

Fig. 2 End result after all osteotomies. The distal femur (red), posterior condyle plane (blue) and proximal tibia plane (green) used for evaluation are shown

orientation and positioning of osteotomy cuts [10,11]. This method uses the preoperative planning CT scan as a reference. The accuracy and reproducibility (test-retest was performed) of the method were below $0.2 \mathrm{~mm}$ for translations and $0.3^{\circ}$ for rotations in the previous technical note. Therefore, differences between the planned and achieved osteotomy, which exceed methodological error, are believed to be caused by transfer errors. Following surgery, a further evaluation CT scan was made. The postoperative CT scan was used to create $3 \mathrm{D}$ polygons, digital models of the tibia and femur. After transforming the femur and tibia polygons into reference images, regions were selected to represent each polygon's cutting plane. A position and a normal vector defined each plane. Several regions on the plane were sampled by automated selection of multiple points within a 3D sphere, positioned within the software (Fig. 3a). The corresponding plane that best fitted the average of these regions was determined and compared in terms of distance and rotational errors to the preoperatively predicted plane (Fig. 3b). Differences in the planned and achieved plane are expressed by the absolute angulation error and the distance error (Fig. 4a). The absolute angulation error is defined as the angle between the normal vectors of the planned and the achieved plane in 3D space. For a better clinical understanding of the difference, these vectors were also projected into the sagittal, coronal and axial planes to evaluate the angular errors in flexion and extension as well as varus and valgus and rotation (Fig. 4b). To this end, anatomical coordinate systems were defined for the femur and the tibia. An extensive explanation of this method and how the coordinate system was defined can be found in Dobbe et al. [11].

\section{Results}

For planes on the tibias, the average displacement error, $d_{\text {err }}$ $( \pm \mathrm{SD})$, of the system was $0.8 \mathrm{~mm}( \pm 0.5 \mathrm{~mm})$. There was an 
Fig. 3 (Left) 3D spherical regions (only one region is shown per osteotomy) (red: posterior femur; yellow: distal femur) chosen for selecting points in the bone model for evaluating the cutting plane orientation. The plane fitted to the osteotomy is shown for the distal femur. (Right) The fitted plane (grey) deviates from the planned plane (blue)

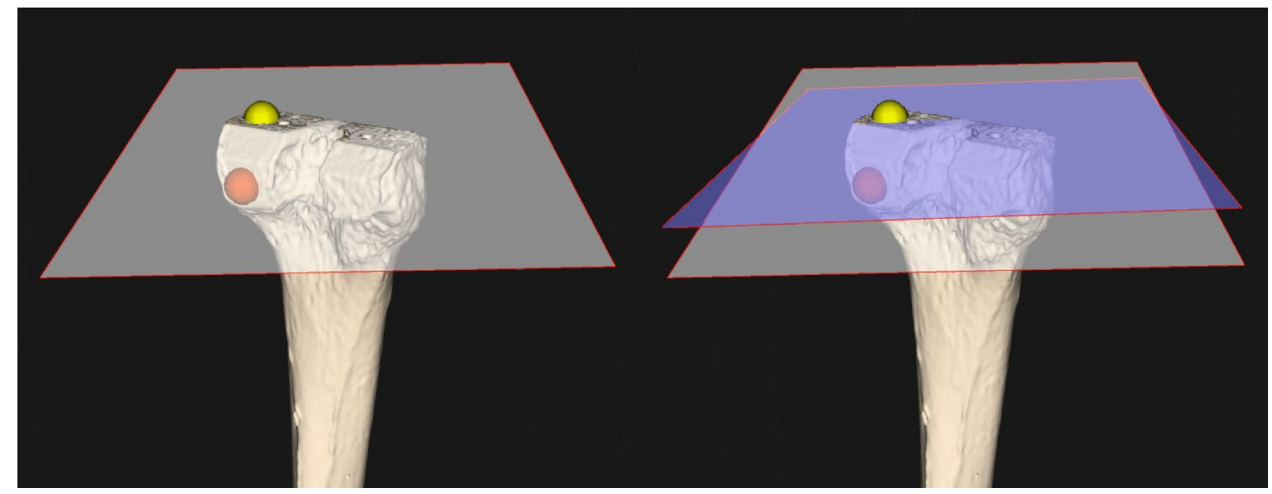

transfer errors were smaller for planes on the tibia than the femur (Tables 1, 2, Fig. 5).

\section{Discussion}

The most important finding of this study is that the discrepancies for the tibia are so small that they can be considered to be clinically irrelevant. The discrepancies for planes on the femur were larger than those on the tibia. For the femurs, we saw systematic discrepancies in the plane orientation towards varus, flexion and external rotation. A combined (absolute error) of $5.2^{\circ}$ is still impressive from a surgical perspective. The varus error, in particular, could change the mechanical axis and, therefore, the placement of the prosthesis. Too little slope might result in a slightly narrow flexion gap. This could be clinically relevant, as this would change the mechanical axis of the leg during walking. The flexion would be less of a problem because the curved shape of the prosthesis. A slight exorotation is preferable as it eases patellar tracking. However, rotational errors are also known to cause clinical complaints so this relevant for some patients. In one specimen (\#6), there was a large error $(>1 \mathrm{~cm})$, with too little bone osteotomized from the distal femur. It is not clear why this difference occurred but could be caused by incorrect placement of the cutting guide or osteotomy block, although the angular errors were not equally large in this specimen. In a live patient, this problem would be apparent and dealt with during surgery as placing the implant would be difficult because the extension gap would be too narrow. It would cause noticeable displacement of the joint line and a larger flexion gap if corrected by decreasing the implant size. Two specimens (\#5 \& \#7) showed a varus error of around $5^{\circ}$ which could produce a clinically relevant change of the mechanical axis of the leg and increased stress on the medial compartment. If the tibial osteotomy also caused too much varus (as in specimen $\# 5$, i.e., $2.6^{\circ}$ of varus) the problem would be exacerbated.

Sawing with the oscillating saw from medial towards the lateral condyle could, in theory, explain the varus 


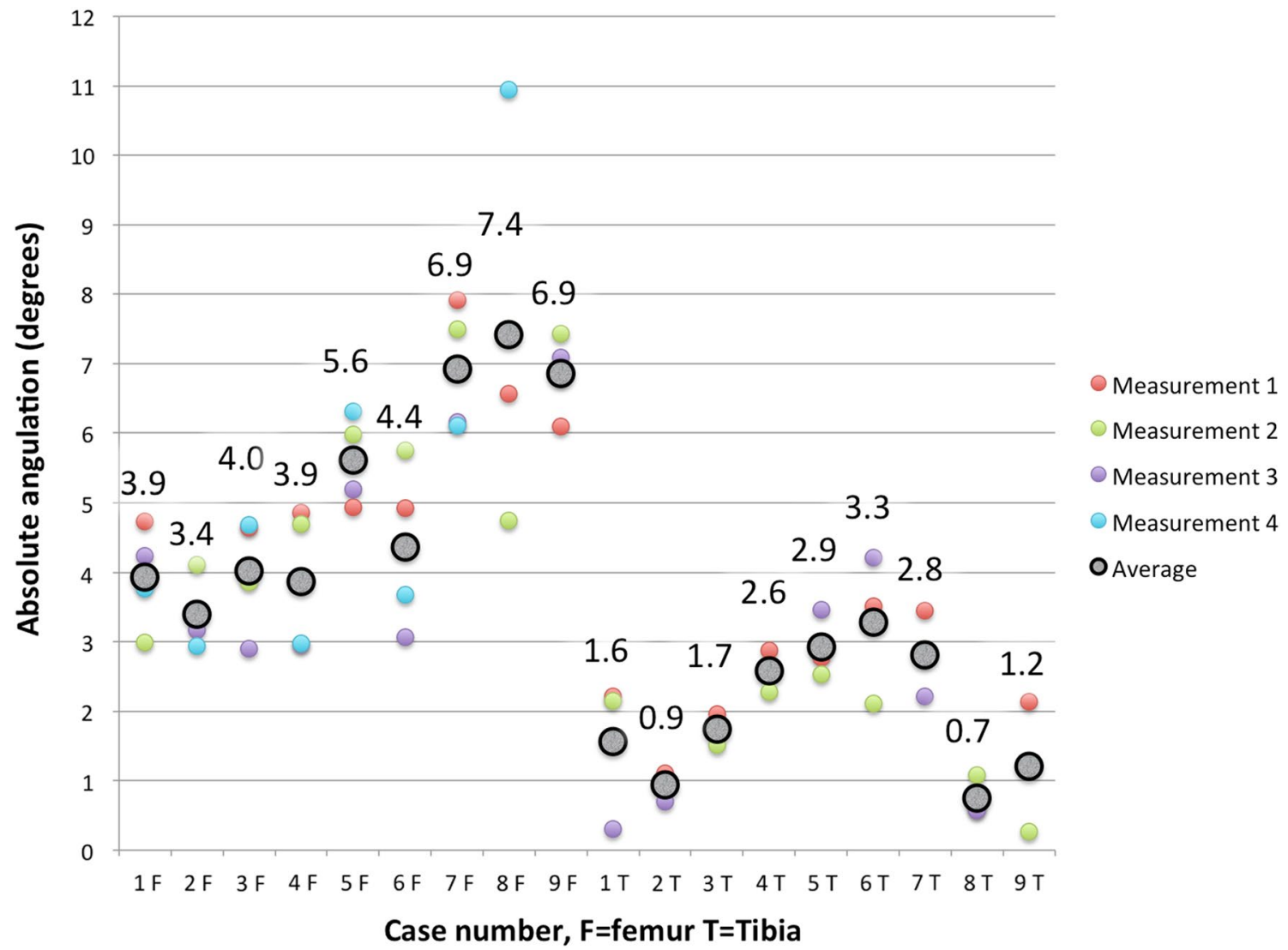

Fig. 5 Absolute angulation difference, combined difference between planned and achieved planes for femur (1F-9F) and tibia (1T-9T). Black circles represent the average differences and colours are the different measurements per knee per osteotomy

Table 1 Separate values of all three local tibial measurements with distance in $\mathrm{mm}$ and angulation errors in ${ }^{\circ}$

\begin{tabular}{lcl} 
Tibia & & \\
\hline $\begin{array}{l}\text { Distance along } Z \\
\text { axis }(\mathrm{mm})\left({ }^{*}\right)\end{array}$ & $\begin{array}{l}\text { Varus }(+) / \\
\text { valgus }(-)\left({ }^{\circ}\right)\end{array}$ & $\begin{array}{l}\text { Flexion }(+) / \\
\text { extension }(-) \\
\left({ }^{\circ}\right)\end{array}$ \\
\hline-0.8 & -0.9 & 1.0 \\
-1.3 & 0.2 & 0.7 \\
-0.6 & 0.5 & -1.5 \\
-1.5 & -1.2 & 2.1 \\
-1.5 & 2.6 & -2.3 \\
0.1 & 0.9 & -3.1 \\
-0.9 & -0.8 & -0.3 \\
-0.5 & 0.6 & 0.4 \\
-0.4 & -0.8 & -0.5 \\
-0.8 & 0.1 & -0.4 \\
0.5 & 1.2 & 1.7
\end{tabular}

The bottom row represents the standard deviation of the error parameters

*Negative values indicate that more bone was removed than intended orientation. Under the assumption that cutting blade deflection increases with the distance from the cutting guide, errors are likely to be largest near the lateral condyle. Furthermore, after the saw blade passes through the medial condyle, it then bridges the intercondylar notch after which it will enter the lateral condyle at a slight angle because of anatomy. This may result in increased deflection of the saw compared to a situation where it enters at a $90-^{\circ}$ angle, as for the tibia. However, after studying the data of our study in detail, it was clear that the most medial sections of the osteotomies show an average varus of $2.5^{\circ}$ and the most lateral sections $2.5^{\circ}$. Therefore, it is unlikely that saw blade deflection is causal to the errors found in this study as the difference between medial and lateral is negligible. It is more likely that asymmetrical positioning of the femur guides would have caused the varus, flexion and external rotation. If the cutting-guide contact points with the femur are slightly higher distally than proximally, for instance because of remaining or overlapping cartilage interposition in the notch, this could result in more varus, flexion and external rotation than desirable. Another explanation could be that producing the guide is easier for the more proximal rounded part of the femur than, for 
Table 2 Separate values of all four local femoral measurements with distance in $\mathrm{mm}$ and angulation errors in $\left(^{\circ}\right)$

\begin{tabular}{lcllr}
\hline & Femur & & \\
\cline { 2 - 5 } & $\begin{array}{l}\text { Distance along } Z \\
\text { axis }(\mathrm{mm})(*)\end{array}$ & $\begin{array}{l}\text { Varus }(+) / \text { valgus } \\
(-) \text { in }\left(^{\circ}\right)\end{array}$ & $\begin{array}{l}\text { Flexion }(-) / \text { exten- } \\
\text { sion }(+) \text { in }\left({ }^{\circ}\right)\end{array}$ & $\begin{array}{l}\text { External }(+) / \text { inter- } \\
\text { nal }(-) \text { rotation } \\
\text { in }\left(^{\circ}\right)\end{array}$ \\
\hline Case 1 & 0.6 & 0.3 & 3.9 & 1.5 \\
Case 2 & -1.6 & -0.6 & -3.0 & 5.0 \\
Case 3 & -1.9 & 2.8 & -2.8 & 1.2 \\
Case 4 & 0.9 & 3.6 & 1.1 & -1.4 \\
Case 5 & 0.3 & 5.7 & 0.1 & 1.2 \\
Case 6 & 10.9 & 3.4 & -2.8 & 4.1 \\
Case 7 & -1.5 & 4.8 & -5.0 & 1.4 \\
Case 8 & 0.6 & 2.1 & -3.4 & 0.7 \\
Case 9 & -1.8 & 3.4 & 1.9 & -1.4 \\
Average & 0.7 & 2.8 & -0.9 & 1.4 \\
SD of cases & 4.0 & 2.0 & 2.7 & 2.2 \\
\hline
\end{tabular}

The bottom row represents the standard deviation of the error parameters

*Negative values indicate that more bone was removed than intended instance, in the notch, which is anatomically more difficult to map.

Most of the previous reports on the accuracy of PSI systems use final implant position as the measure to judge positioning accuracy $[6,8,15,17,18,23]$. Nam et al. [21] compared 41 knees implanted using CAS with 41 knees implanted using the Signature MRI-based PSI method. They noted that in the Signature PSI group, $88 \%$ of tibial components had an alignment within $2^{\circ}$ perpendicular to the neutral mechanical axis. For the femoral components, $90 \%$ had an alignment within $2^{\circ}$ perpendicular to the femur mechanical axis. Their results are better than the results reported in our study, but the numbers are difficult to compare, as they did not evaluate the osteotomies. In their study, the prosthesis orientation was measured by hand on plane radiographs, and no measurement error was given for the evaluation method. In a study by $\mathrm{Ng}$ et al. [22], 569 implants using Signature were reviewed retrospectively. Again the position of the implant was evaluated using long-leg radiographs. It was reported that the mechanical axis passed through the central third of the knee more often with Signature PSI (88\%) than with manual instrumentation (78\%). Furthermore, they reported that PSI had $10 \%$ outliers $\left(>2^{\circ}\right)$ for the tibial component and $22 \%$ for the femoral component. The finding that the femoral orientation is less accurate is consistent with our study. A third study used postoperative CT to evaluate the Signature system in 23 TKA patients [24], but they only reported femoral implant rotation about the long axis. This study also used final implant position as a measure of success and also omits reporting the measurement error of the evaluation method and the variability in their observations. They did see median postoperative rotation of $0^{\circ}$ for the femur as planned.
Some previous studies have tried to assess the transfer accuracy of other PSI systems more accurately by using computer navigation to assess the position and orientation of the cutting guide $[7,20]$. Conteduca et al. reported that for 12 procedures the mean deviation of the tibial guide from the ideal alignment on the coronal plane was $1.2^{\circ}\left( \pm 1.5^{\circ}\right)$ and in the sagittal plane $3.8^{\circ}\left( \pm 2.4^{\circ}\right)$ [7]. On the coronal plane, the mean deviation of the femoral guide from the ideal alignment was $1.2^{\circ}\left( \pm 0.6^{\circ}\right)$ and in the sagittal plane was $3.7^{\circ}\left( \pm 2^{\circ}\right)$. Lustig et al. reported that for 60 procedures, the mean deviation of the tibial guide from the ideal alignment on the coronal plane was $0.6^{\circ}\left( \pm 1.9^{\circ}\right)$ and in the sagittal plane $-0.1^{\circ}\left( \pm 2.6^{\circ}\right)$ [20]. On the coronal plane, the mean deviation of the femoral guide from the ideal alignment was $0.2^{\circ}\left( \pm 1.8^{\circ}\right)$ and in the sagittal plane was $2.1^{\circ}$ $\left( \pm 2.8^{\circ}\right)$. No researchers investigated the system studied in this paper. The results described in these studies seem to corroborate the data reported in our study. However, computer navigation has been known to result in displacement errors of up to $2 \mathrm{~mm}$ caused by the effect of the distance between the stereoscopic camera system and surgical tools [27]. Furthermore, the computer navigation approach does not take into account that the achieved osteotomy might not have a direct relationship with the guide because of the saw blade deflection mentioned previously or changes made on basis of clinical judgment after placing the guides. Changes might also occur with the removal of the pin-positioning guides and placement of the saw guide. Therefore, the position of the guide might not actually be related to achieving the actual planned plane. Thus, the above-mentioned studies are not equipped to evaluate the transfer accuracy of the osteotomy plane itself. It could be hypothesized that cutting block guides produced with a slit to guide the saw directly 
are more accurate than pin-positioned guides because the first technique avoids an extra intraoperative step. One study reported on the mean discrepancies of distal femoral and proximal tibial cuts using the Visionaire systems, a cutting block-guided system, by measuring the thickness of the removed bone segment and comparing it to the planned values [28]. The mean discrepancy was reported to be $3.1 \pm 1.0$ and $3.1 \pm 1.1 \mathrm{~mm}$ for distal femoral medial end lateral cuts, respectively, and $2.7 \pm 0.9 \mathrm{~mm}$ for both proximal tibial medial and lateral cuts. In our study, an average discrepancy of $0.7 \pm 4.0 \mathrm{~mm}$ for femoral cuts and $0.8 \pm 0.5 \mathrm{~mm}$ for tibial cuts was found. Therefore, it seems that using a cutting block guide does not result in fewer discrepancies. However, it is arguable that the two measuring techniques are too diverse to make this comparison.

The accuracy and reproducibility of the method used in our study were below $0.2 \mathrm{~mm}$ for translations and $0.3^{\circ}$ for rotations in the previous technical note [11]. Therefore, differences between planned and achieved osteotomies exceeding the methodological error are expected to be caused by transfer errors.

In the here reported study, CT scan data was used to plan and evaluate the osteotomy planes because CT scans yield high bone-soft tissue contrast which makes it easier to assess the transfer accuracy of the osteotomy planes. Therefore, our results cannot be applied to the MRI version of the Signature system. Further studies should systematically compare both CT and MRI systems to confirm which yields more accurate results. The downside of a guide produced on the basis of CT data is that it needs supporting points that lie outside the cartilage layer. MRI-produced guides sit adjacent to the cartilage layer and have a larger surface area to ensure adequate placement. However, MRI-produced guides have been shown to be less accurate than CT-produced guides in other systems [26].

There are some limitations to this study. The system currently studied is a pin-positioning guide. After placements of the pins, the guide needs to be replaced by a standard cutting block. This potentially introduces the risk of pin movement and thus decreased accuracy. Guides with a slotted saw blade sleeve could potentially be more accurate. Furthermore, this is a cadaveric study so the clinical effects and outcome cannot be measured and the results may not be transferrable to real-life total knee replacement surgery. Not all the cadavers had arthritic knees, so the positioning of the guides might be less accurate on osteophytic bones in a live patient. To position the guide for correct cutting, any soft tissue trapped between bone and guide could alter the orientation of the guide. In cadaver limbs, any obstructing soft tissues can simply be cut away and the guide positioned on the bone accurately. However, for patients, it is important to cause as little soft tissue damage as possible during surgery as this can impair the recovery process. Nevertheless, great care was taken to perform the operations as if on a live patient. It would have been preferable to analyse a larger number of specimens but the cost aspect limited us to a restricted number of specimens. However, the size of the study group is, in part, compensated by the highly accurate evaluation technique. Cost also prevented us from actually placing expensive implants so prosthesis positioning could not be evaluated. This has been studied by several other authors and was not the main focus of the here reported study. Finally, we only evaluate the most commonly used system (Signature) so the validity of this study for other systems is not necessarily transferrable.

\section{Conclusion}

The production of guides produced by means of a 3D system based on CT data was assessed on cadaver specimen knees. The predicted osteotomy planes were more accurate for the tibia than for the femur. The use of 3D system techniques in TKA surgery provides accurate intraoperative guidance tailored to individual patients ensuring better placement of the implant, even for patients with bone deformities. Future studies could investigate further benefits such as reduced operation time, potentially fewer complications and longer implant survival with this method of controlled and improved component alignment.

Acknowledgements We wish to acknowledge the efforts of the pathology department of the AMC and the department of anatomy and embryology of the AMC in their support needed to perform this study. Furthermore, we wish to acknowledge the support provided by Biomet by supplying us with the materials and support necessary to perform this study. Lastly, we would like to thank P.A. Kievit-Tyson for her editing work on the manuscript.

\section{Compliance with ethical standards}

Conflict of interest The authors declare that they have no conflict of interest.

Funding Biomet (Biomet, Inc, Warsaw, Ind) supplied the department of orthopaedic surgery of the Academic Medical Center Amsterdam with an unrestricted research grant for the employment of a $\mathrm{PhD}$ student.

Ethical approval Institutional review board approval was deemed not necessary as the study involved anonymous human cadaveric material as acquired with permission in accordance with the Dutch donor system.

Open Access This article is distributed under the terms of the Creative Commons Attribution 4.0 International License (http://creativecommons.org/licenses/by/4.0/), which permits unrestricted use, distribution, and reproduction in any medium, provided you give appropriate credit to the original author(s) and the source, provide a link to the Creative Commons license, and indicate if changes were made. 


\section{References}

1. Arora J, Sharma S, Blyth M (2005) The role of pre-operative templating in primary total knee replacement. Knee Surg Sport Traumatol Arthrosc 13:187-189

2. Aslam N, Lo S (2005) Pre-operative planning and the role of templating in total knee arthroplasty. J Eval Clin Pract 11:93-94

3. Aslam N, Lo S, Nagarajah K, Pasapula C, Akmal M (2004) Reliability of preoperative templating in total knee arthroplasty. Acta Orthop Belg 70:560-564

4. Barrack RL, Ruh EL, Williams BM, Ford AD, Foreman K, Nunley RM (2012) Patient specific cutting blocks are currently of no proven value. J Bone Joint Surg Br 94:95-99

5. Bauwens K, Matthes G, Wich M, Gebhard F, Hanson B, Ekkernkamp A et al (2007) Navigated total knee replacement. A meta-analysis. J Bone Joint Surg Am 89:261-269

6. Chareancholvanich K, Narkbunnam R, Pornrattanamaneewong C (2013) A prospective randomised controlled study of patientspecific cutting guides compared with conventional instrumentation in total knee replacement. Bone Joint J 95-B:354-359

7. Conteduca F, Iorio R, Mazza D, Caperna L, Bolle G, Argento G et al (2013) Evaluation of the accuracy of a patient-specific instrumentation by navigation. Knee Surg Sport Traumatol Arthrosc 21:2194-2199

8. Daniilidis K, Tibesku CO (2013) Frontal plane alignment after total knee arthroplasty using patient-specific instruments. Int Orthop 37:45-50

9. Delp SL, Stulberg SD, Davies B, Picard F, Leitner F (1998) Computer assisted knee replacement. Clin Orthop Relat Res 354:49-56

10. Dobbe JG, Strackee SD, Schreurs AW, Jonges R, Carelsen B, Vroemen JC et al (2011) Computer-assisted planning and navigation for corrective distal radius osteotomy, based on pre- and intraoperative imaging. IEEE Trans Biomed Eng 58:182-190

11. Dobbe JGG, Kievit AJ, Schafroth MU, Blankevoort L, Streekstra GJ (2014) Evaluation of a CT-based technique to measure the transfer accuracy of a virtually planned osteotomy. Med Eng Phys 36:1081-1087

12. Fang D, Ritter MA (2009) Malalignment: forewarned is forearmed. Orthopedics 32:1

13. Hafez MA, Chelule KL, Seedhom BB, Sherman KP (2006) Computer-assisted total knee arthroplasty using patient-specific templating. Clin Orthop Relat Res 444:184-192

14. Heal J, Blewitt N (2002) Kinemax total knee arthroplasty: trial by template. J Arthroplasty 17:90-94

15. Heyse TJ, Tibesku CO (2014) Improved femoral component rotation in TKA using patient-specific instrumentation. Knee 21:268-271
16. Howcroft DW, Fehily MJ, Peck C, Fox A, Dillon B, Johnson DS (2006) The role of preoperative templating in total knee arthroplasty: comparison of three prostheses. Knee 13:427-429

17. Kerens B, Boonen B, Schotanus M, Kort N (2013) Patient-specific guide for revision of medial unicondylar knee arthroplasty to total knee arthroplasty. Acta Orthop 84:165-169

18. Klatt BA, Goyal N, Austin MS, Hozack WJ (2008) Custom-fit total knee arthroplasty (OtisKnee) results in malalignment. J Arthroplasty 23:26-29

19. Krishnan SP, Dawood A, Richards R, Henckel J, Hart AJ (2012) A review of rapid prototyped surgical guides for patient-specific total knee replacement. J Bone Joint Surg Br 94:1457-1461

20. Lustig S, Scholes CJ, Oussedik SI, Kinzel V, Coolican MR, Parker DA (2013) Unsatisfactory accuracy as determined by computer navigation of VISIONAIRE patient-specific instrumentation for total knee arthroplasty. J Arthroplasty 28:469-473

21. Nam D, Maher PA, Rebolledo BJ, Nawabi DH, McLawhorn AS, Pearle AD (2013) Patient specific cutting guides versus an imageless, computer-assisted surgery system in total knee arthroplasty. Knee 20:263-267

22. Ng VY, DeClaire JH, Berend KR, Gulick BC, Lombardi AV Jr (2012) Improved accuracy of alignment with patient-specific positioning guides compared with manual instrumentation in TKA. Clin Orthop Relat Res 470:99-107

23. Nunley RM, Ellison BS, Zhu J, Ruh EL, Howell SM, Barrack RL (2012) Do patient-specific guides improve coronal alignment in total knee arthroplasty? Clin Orthop Relat Res 470:895-902

24. Silva AF, Sampaio RF, Pinto E (2014) Patient-specific instrumentation improves tibial component rotation in TKA. Knee Surg Sports Traumatol Arthrosc 22:636-642

25. Thienpont E, Bellemans J, Delport H, Van Overschelde P, Stuyts B, Brabants K et al (2013) Patient-specific instruments: industry's innovation with a surgeon's interest. Knee Surg Sport Traumatol Arthrosc 21:2227-2233

26. White D, Chelule KL, Seedhom BB (2008) Accuracy of MRI vs CT imaging with particular reference to patient specific templates for total knee replacement surgery. Int J Med Robot 4:224-231

27. Wittmann W, Wenger T, Loewe E, Lueth TC (2011) Official measurement protocol and accuracy results for an optical surgical navigation system (NPU). Conf Proc IEEE Eng Med Biol Soc 2011:1237-1240

28. Zambianchi F, Colombelli A, Digennaro V, Marcovigi A, Mugnai R, Fiacchi F et al (2017) Assessment of patient-specific instrumentation precision through bone resection measurements. Knee Surg Sport Traumatol Arthrosc 25:2841-2848 